\title{
QUESTIONS AROUND THE THUE-MORSE SEQUENCE
}

\author{
Martine QuefFÉLEC
}

Dedicated to the memory of Pierre Liardet

\begin{abstract}
We intend to unroll the surprizing properties of the Thue-Morse sequence with a harmonic analysis point of view, and mention in passing some related open questions.
\end{abstract}

\section{Communicated by Jean-Louis Verger-Gaugry}

P i e r r e L i a r d et has been highly interested in symbolic dynamical systems associated to expansions of real numbers or automatic sequences, but also in distribution and uniform distribution of sequences, and in many other related questions. More precisely, he paid much attention to the classification of dynamical systems, those arising as an extension of some discrete system ( $q$-multiplicative sequences, Ostrovski's sequence), and he encountered this way cocycle equations; he also studied the property of disjointness for dynamical systems (due to F u r s t e n b e r g), with emphasis on number theoretical systems, by relaying the notion of disjoint sequences developed by $\mathrm{K}$ a $\mathrm{m}$ a e; this notion is central in Sarnak's conjecture, and recent progress has been done by B o u r ga in, S a r n a k and $\mathrm{Z}$ i e g l e r, also by F e r e n c zi, M a u d u it, Rivat ... involving the Thue-Morse sequence together with the Moebius function, and making use of the associated trigonometric polynomials; for a long time, Pierre has devoted himself to bad distributions of sequences and quantitative aspects of this question, with estimates for the discrepancy and description of bounded remainder sets.

I don't forget many sharp contributions he published on numeration systems and continued fractions, but I chose to give an overview on the amazing properties of the topical Thue-Morse sequence, which lends itself very well to computations, with some remaining questions. It is also after a question of Pierre on the Thue-Morse correlation that we met and became friends.

Keyw ords: Thue-Morse, Rudin-Shapiro, sum of digits, trigonometric polynomial, correlation measure, singular measure. 


\section{Introduction and progression}

I consider the Thue-Morse sequence (TM) on the \pm 1 -alphabet which is defined by

$$
\mathbf{t}=\left(t_{n}\right):=\left((-1)^{S_{2}(n)}\right), \quad \text { with } \quad S_{2}(n)
$$

the sum of the 2-digits of the integer $n$; its properties are well-known: $\mathbf{t}$ is a non-periodic, 2-multiplicative $\left(t_{2^{n} a+b}=t_{a} t_{b}, a \geq 0,0 \leq b<2^{n}\right)$, 2-automatic sequence with 0 -mean. It enjoys quite a few extremal properties and, all those informations can be derived from the quite simple recursive definition

$$
t_{2 n}=t_{n}, \quad t_{2 n+1}=-t_{n}, \quad t_{0}=1 .
$$

Despite this elementary symmetry property, many questions about it are widely open and some others are still in progress, including generalizations to other sequences, such as $\left(e^{2 i \pi \alpha S_{2}(n)}\right)$ or Rudin-Shapiro sequences, more generally $q$ -multiplicative and $q$-automatic sequences.

The aim of this survey is to give an overview of these fascinating properties and to put together scattered existing results, with quite natural resulting questions. We shall follow the Ariane's thread, more or less historically coherent: from sub-sequences to polynomial estimates, from polynomials to correlation measure and then back to sub-sequences. More about history of this sequence can be found in 22 .

We denote by $\left(P_{N}\right)$ and $\left(p_{n}\right)$ the Thue-Morse polynomials defined by

$$
P_{N}(z)=\sum_{k<N} t_{k} z^{k}, \quad p_{n}=P_{2^{n}}, \quad p_{0}=1,
$$

so that (11) gives, when restricted to $|z|=1$,

$$
\left|P_{N}(z)\right| \leq \sum_{n=1}^{r}\left|p_{n}(z)\right|, \quad r=\left[\log _{2} N\right] .
$$

From (11) again, we get

$$
p_{n+1}(z)=(1-z) p_{n}\left(z^{2}\right),
$$

leading to $p_{n+1}(z)=\prod_{j=0}^{n}\left(1-z^{2^{j}}\right)$, a factorization that appears under a more general form in the study of $q$-multiplicative sequences. The recursive relations (3) can be gathered into a single one through the generating function

$$
F(z)=\sum_{n \geq 0} t_{n} z^{n}=(1-z) F\left(z^{2}\right)=\cdots=\prod_{j \geq 1}\left(1-z^{2^{j}}\right)
$$




\section{QUESTIONS AROUND THE THUE-MORSE SEQUENCE}

but the sequence of trigonometric polynomials $\left(p_{n}\right)$ plays a crucial role in the dynamical study of the TM sequence as we shall see.

From now on, the circle $\mathbb{T}$ is identified with $[0,1)$ and we make use of the classical notation: $e(t)=e^{2 \pi i t}$. We denote by the same symbol $\left(p_{n}\right)$ the Thue-Morse trigonometric polynomials:

$$
p_{n}(t):=p_{n}(e(t))
$$

so that

$$
p_{n+1}(t)=\prod_{j=0}^{n}\left(1-e\left(2^{j} t\right)\right)
$$

Concerning the uniform norm of $p_{n}$, a first estimate can easily be obtained from (4); actually,

$$
\left|p_{n}(t)\right|=\prod_{j=0}^{n-1} 2\left|\sin \left(\pi 2^{j} t\right)\right|
$$

so that, by grouping terms 2 by 2 in (5), and making use of the elementary inequality

$$
|\sin x \sin (2 x)| \leq 4 / 3 \sqrt{3} \quad \text { for every } x
$$

we deduce

$$
\left\|p_{n}\right\|_{\infty} \leq 2^{n}\left(\frac{4}{3 \sqrt{3}}\right)^{n / 2}=2^{n(1-\eta)} \quad \text { with } \quad \eta=\log (3 \sqrt{3}) / \log 4-1 \sim 0,18
$$

Actually, a tricky observation of G elfond [14] leads to the sharp estimate:

Theorem 1.1 (Gelfond). For every $n \geq 1$,

$$
\sup _{t \in[0,1]}\left|p_{n}(t)\right| \leq \frac{2}{\sqrt{3}} 2^{n \log 3 / \log 4}
$$

Moreover, if $\omega$ is some primitive cubic root of the unity,

$$
\left|1-\omega^{k}\right|=\sqrt{3} \quad \text { for all } \quad k \neq \equiv 0 \bmod 3 ;
$$

from this,

$$
\sup _{t \in[0,1]}\left|p_{n}(t)\right| \geq\left|p_{n}(\omega)\right|=\prod_{k=0}^{n-1}\left|1-\omega^{2^{k}}\right|=3^{n / 2}=2^{n \log 3 / \log 4} .
$$

Together with (2), this gives the uniform exponent $\log 3 / \log 4 \sim 0.7925$. 


\section{First questions on the TM sequence}

We begin this survey by two old, but still very active, subjects with recent improvements involving the $L^{1}$-estimate of the TM polynomials sequence, more powerful than the $L^{\infty}$-one: subsequences and discrepancy.

\subsection{Subsequences}

One of the first questions we consider about this sequence concerns the behavior of specific subsequences. After D. J. N e w m an [25] and J. C oquet 6], J. M. D u m o n t [1] studied the restriction of $\left(t_{n}\right)$ to an arithmetic progression (cf. also [35]) but the problem of dealing with a zero density sequence of indices turned out to be much more difficult. For example, the study of the subsequences $\left(S_{2}(p)\right)_{p \in \mathcal{P}}, \mathcal{P}$ the set of prime numbers, and $\left(S_{2}\left(n^{2}\right)\right)_{n \geq 1}$ of the sum of digits has been achieved quite recently with the following precise results.

Improving a theorem from Fouvry-Mauduit [13, Mauduit-Ri$\mathrm{v}$ at [23] proved

Theorem 2.1 (Mauduit-Rivat 2010). For any $\alpha \in \mathbb{R} \backslash \mathbb{Z}$, one can find $\sigma(\alpha)>0$ such that

$$
\sum_{\substack{p \leq x \\ p \in \mathcal{P}}} e\left(\alpha S_{2}(p)\right) \ll x^{1-\sigma(\alpha)}, \quad \text { for } \quad x \geq 2 .
$$

As a corollary, by taking $\alpha=1 / 2$, we get that the TM sequence has a zero mean on the prime numbers: $\lim _{N \rightarrow \infty} \frac{1}{\pi(N)} \sum_{p \leq N, p \in \mathcal{P}} t_{p}=0$. (Before this estimate, the existence of infinitely many either even or odd numbers in the sequence $S_{2}(p)$ was an open question.)

Two years later (though the article has been published before), with additional technics since the sequence of square numbers is more sparse than the prime sequence, they proved [24]:

Theorem 2.2 (Mauduit-Rivat 2009). For any $\alpha \in \mathbb{R} \backslash \mathbb{Z}$, one can find $c(\alpha)>0$ et $x_{0}$ such that

$$
\sum_{n \leq x} e\left(\alpha S_{2}\left(n^{2}\right)\right) \ll x^{1-c(\alpha)}\left(1+\log _{2} x\right)^{5}, \quad \text { for } \quad x \geq x_{0} .
$$

In particular, the TM sequence has a zero mean when restricted to the square numbers:

$$
\lim _{N \rightarrow \infty} \frac{1}{N} \sum_{n \leq N} t_{n^{2}}=0
$$

A similar result is also proved for the sum of $q$-digits,

$$
q \geq 2 \quad \text { as long as } \quad(q-1) \alpha \notin \mathbb{Z} \text {. }
$$




\section{QUESTIONS AROUND THE THUE-MORSE SEQUENCE}

The question of

$$
\lim _{N \rightarrow \infty} \frac{1}{N} \sum_{n \leq N} t_{3^{n}}
$$

seems to be hard and could be related to the uniform distribution modulus 1 of $(3 / 2)^{n}[19]$.

The asymptotic behavior of the $L^{1}$-norm of the TM polynomials proves to be relevant in the work of Fouvry-Mauduit (behind the first quoted theorem) and they make it clear through considerations on the associated Perron-Frobenius operator (defined later).

\subsection{Discrepancy}

We denote by $n_{1}<n_{2}<\cdots<n_{k}<\cdots$ the occurrence indices of 1 in the sequence $\left(t_{n}\right)$. The distribution modulo 1 of the sequence $\left(n_{k} \alpha\right)$ has been studied in the sixties and the set of all $\alpha$ for which the sequence $\left(n_{k} \alpha\right)$ is uniformly distributed has been identified to $\mathbb{R} \backslash \mathbb{Q}$. In a recent article [1, A istleitner, Hofer \& L a r c her analyze in a deep way the discrepancy of $\left(n_{k} \alpha\right)$ according to $\alpha \in \mathbb{R}$ and once more reveal the role played by the $L^{1}$-norm of the TM polynomials.

Recall that, for any sequence $\left(u_{n}\right) \subset[0,1]$,

$$
D_{N}^{*}\left(u_{1}, \ldots, u_{N}\right)=\sup _{a \in[0,1]}\left|\frac{1}{N} \sum_{1 \leq n \leq N} \mathbf{1}_{[0, a]}\left(u_{n}\right)-a\right|
$$

is the uniform discrepancy of the finite sequence $u_{1}, \ldots, u_{N}$. Their result goes as follows:

TheOREM 2.3. Let be $\varepsilon>0$. For almost all $\alpha \in \mathbb{R}$,

$$
\begin{aligned}
& N D_{N}^{*}\left(\left\{n_{1} \alpha\right\}, \ldots,\left\{n_{N} \alpha\right\}\right)=O\left(N^{1+\log _{2} \lambda+\varepsilon}\right), \\
& N D_{N}^{*}\left(\left\{n_{1} \alpha\right\}, \ldots,\left\{n_{N} \alpha\right\}\right) \geq\left(N^{1+\log _{2} \lambda-\varepsilon}\right),
\end{aligned}
$$

where $\lambda>2$ depends on the $L^{1}$-norm of $p_{n}$ :

$$
2^{-N}\left\|p_{N}\right\|_{1}=\lambda^{N}(1+o(1))
$$

in other words,

$$
1+\log _{2} \lambda=\beta \quad \text { if } \quad\left\|p_{N}\right\|_{1} \approx 2^{N \beta}
$$

The proof combines Koksma's inequality together with Erdős-Turan's one. 


\section{3. $L^{1}$-norm of the TM polynomials}

We are thus led to this question: how to describe the asymptotic behaviour of $L^{1}$-norm of the TM polynomials? Starting with (5): $\left|p_{n}(t)\right|=\prod_{j=0}^{n-1} 2\left|\sin \left(\pi 2^{j} t\right)\right|$, we suggest sharper and sharper tricks for this estimate.

\subsection{Interpolation method}

Let us recall briefly the interpolation method: if $p, q, r>0$ are such that $1 / q=\theta / p+(1-\theta) / r$ with $0 \leq \theta \leq 1$, then we have

$$
\|P\|_{q} \leq\|P\|_{p}^{\theta}\|P\|_{r}^{(1-\theta)} \text {. }
$$

- The interpolation inequality $L^{1}-L^{2}-L^{\infty}$, i.e., $\|P\|_{2}^{2} \leq\|P\|_{1}\|P\|_{\infty}$, produces some lower bound for the $L^{1}$-norm, provided that an upper bound is known for $\left\|P_{N}\right\|_{\infty}:\left\|P_{N}\right\|_{1} \geq\left\|P_{N}\right\|_{2}^{2} /\left\|P_{N}\right\|_{\infty}=N /\left\|P_{N}\right\|_{\infty}$.

But, as observed in the introduction, $\left\|p_{n}\right\|_{\infty} \sim 2^{n \log 3 / \log 4}$, and this interpolation inequality cannot give a better estimate than

$$
\left\|p_{n}\right\|_{1} \gtrsim 2^{n \epsilon} \quad \text { with } \quad \epsilon=1-\log 3 / \log 4 \sim 0.2075 \ldots
$$

- We get a better lower bound by using the interpolation $L^{1}-L^{2}-L^{4}$, and this is all the more interesting as an accurate computation of the $L^{4}$-norm can be performed.

Proposition 3.1. We have: $\left\|p_{n}\right\|_{4}^{2} \approx 2^{n \gamma}$ with $\gamma=\log _{2}(1+\sqrt{17}) / 2$.

Corollary 3.1. As a consequence, $\left\|p_{n}\right\|_{1} \gtrsim 2^{n \beta}$ with $\beta \sim 0.3215 \ldots$

Proof. We begin with the estimate of the $L^{4}$-norm which will be used later. We consider the normalized polynomial $q_{N}:=2^{-N}\left|p_{N}\right|^{2}$ with spectrum into ] $-2^{N}, 2^{N}$ [; by the previous inequality (5]),

$$
q_{N}(t)=\prod_{n<N}\left(1-\cos \left(2 \pi 2^{n} t\right)\right)=: \sum_{|k|<2^{N}} a_{k} e(k t) .
$$

Now, Parseval's inequality takes the following form:

and we are just led to estimate

$$
\left\|p_{N}\right\|_{4}^{4}=\left\|2^{N} q_{N}\right\|_{2}^{2}=2^{2 N} \sum_{|k|<2^{N}}\left|a_{k}\right|^{2}
$$

$$
\sigma_{N}:=\sum_{|n|<2^{N}}\left|a_{n}\right|^{2} .
$$




\section{QUESTIONS AROUND THE THUE-MORSE SEQUENCE}

By identifying the coefficients in the identity:

$$
\sum_{|k|<2^{N}} a_{k} e(k t)=(1-\cos 2 \pi t) \sum_{|k|<2^{N-1}} a_{k} e(2 k t),
$$

we can easily check the induction relation satisfied by the coefficients $a_{n}$ :

$$
a_{2 k}=a_{k}=a_{-k}, \quad a_{2 k+1}=-\frac{1}{2}\left(a_{k}+a_{k+1}\right), \quad \text { for } k \geq 0 .
$$

A first estimate follows:

LEMMA 3.1. We have $\sum_{|n|<2^{N}}\left|a_{n}\right|^{2} \approx \kappa^{N}=2^{N \log _{2} \kappa}$, where

$$
\kappa=\frac{1+\sqrt{17}}{4} .
$$

P r o o f. We have to estimate $\sigma_{N}:=\sum_{|n|<2^{N}}\left|a_{n}\right|^{2}$ and we first establish a relation satisfied by the $\left(\sigma_{N}\right)$ (up to $O(1)$ ), deduced from (6) $)$.

$$
\sum_{|n|<2^{N+1}}\left|a_{n}\right|^{2}=\sum_{|n|<2^{N}}\left|a_{2 n}\right|^{2}+\left|a_{2 n+1}\right|^{2}=\sum_{|n|<2^{N}}\left|a_{n}\right|^{2}+\frac{1}{4} \sum_{|n|<2^{N}}\left|a_{n}+a_{n+1}\right|^{2} ;
$$

so that, by expanding the square,

$$
\sigma_{N+1}=\sigma_{N}+\frac{1}{4} \sigma_{N}+\frac{1}{4} \sigma_{N}+\frac{1}{2} \sum_{|n|<2^{N}} a_{n} a_{n+1} .
$$

In this last sum, dealing first with non negative $n$, we separate once more odd and even indices to get via (6)

$$
\begin{aligned}
\sum_{n<2^{N}} a_{n} a_{n+1} & =\sum_{n<2^{N-1}} a_{2 n} a_{2 n+1}+\sum_{n<2^{N-1}} a_{2 n+1} a_{2 n+2} \\
& =\sum_{n<2^{N-1}} a_{2 n+1}\left(a_{2 n}+a_{2 n+2}\right)=\sum_{n<2^{N-1}} a_{2 n+1}\left(a_{n}+a_{n+1}\right) \\
& =\sum_{n<2^{N-1}} a_{2 n+1}\left(-2 a_{2 n+1}\right) \\
& =-2 \sum_{n<2^{N-1}} a_{2 n+1}^{2}=-2\left(\sum_{n<2^{N}} a_{n}^{2}-\sum_{n<2^{N-1}} a_{2 n}^{2}\right) \\
& =-2\left(\sigma_{N}-\sigma_{N-1}\right) .
\end{aligned}
$$

Plugging this identity into (8) and using $a_{n}=a_{-n}$, we finally derive a linear induction formula (up to $O(1)$ ) for $\sigma_{N}$ :

$$
\sigma_{N+1}=\frac{1}{2} \sigma_{N}+\sigma_{N-1}
$$


Hence the expected behavior, with some constant $c$,

$$
\sum_{|n|<2^{N}}\left|a_{n}\right|^{2}=c \kappa^{N}+O(1)=c\left(\frac{1+\sqrt{17}}{4}\right)^{N}+O(1)
$$

since $\kappa$ is given as a solution of $2 x^{2}-x-2=0$.

Turning back to the $p_{N}$, we obtain

$$
\left\|p_{N}\right\|_{4}^{4}=2^{2 N} \sum_{|n|<2^{N}}\left|a_{n}\right|^{2} \sim c 2^{2 N} \kappa^{N}
$$

and the proposition

$$
\left\|p_{N}\right\|_{4}^{2} \approx 2^{N} \kappa^{N / 2}=2^{N \gamma}
$$

if

$$
\gamma=1+\left(\log _{2} \kappa\right) / 2=\left[\log _{2}(1+\sqrt{17})\right] / 2
$$

Pr o of. We now deduce the corollary.

Since

$$
1 / 2=1 / 3 \cdot 1+2 / 3 \cdot 1 / 4
$$

the interpolation method leads to the following inequality:

$$
\|P\|_{2} \leq\|P\|_{1}^{1 / 3}\|P\|_{4}^{2 / 3}
$$

whence

$$
\|P\|_{1} \geq\|P\|_{2}^{3} /\|P\|_{4}^{2}
$$

and the $L^{4}$-norm estimate for $p_{n}$ gives

$$
\left\|p_{n}\right\|_{1} \geq\left\|p_{n}\right\|_{2}^{3} /\left\|p_{n}\right\|_{4}^{2} \gg 2^{3 n / 2} \cdot 2^{-n} \kappa^{-n / 2}=2^{n \beta}
$$

with

$$
\beta=\frac{1}{2} \log _{2}\left(\frac{8}{1+\sqrt{17}}\right) \sim 0,3215
$$

Remark. The so-obtained estimates are not optimal and it seems that even more general interpolation inequalities could not lead to the expected behavior. However the following question remains: can we estimate $\left\|p_{n}\right\|_{2 k}$ for any $k \geq 1$ ? This question has been investigated with some recent results for the RudinShapiro polynomials [25, 10, 30]. 


\section{QUESTIONS AROUND THE THUE-MORSE SEQUENCE}

\subsection{Transfer operator}

The right point of view, exploited by Fouvry and Mauduit (1996) and A is t leitner, Hofer and Larcher (2015), consists in a functional interpretation of these $L^{1}$-norms: $\left\|p_{n}\right\|_{1}$, by introducing the so-called transfer operator that we describe in this context.

Putting $f_{n}(t)=\left|p_{n}(t)\right|$, we have by (4)

$$
f_{n}(t)=2|\sin \pi t|\left|p_{n-1}(2 t)\right|=2|\sin \pi t| f_{n-1}(2 t)
$$

and we are led to consider the weighted composition operator $\mathcal{L}$ on $L^{1}(0,1)$ defined by

$$
\mathcal{L}(f)=w \cdot f \circ \sigma
$$

where $\sigma$ denotes the 2-shift $x \mapsto 2 x \bmod 1$ and the weight $w(t)$ is $2|\sin \pi t|$. With this tool,

$$
f_{n}=\mathcal{L} f_{n-1}=\cdots=\mathcal{L}^{n} f_{0}=\mathcal{L}^{n} \mathbf{1}
$$

and the $L^{1}$-norms of $\left(p_{n}\right)$ are nothing but

$$
u_{n}:=\int_{0}^{1} f_{n}(t) \mathrm{d} t=\int \mathcal{L}^{n} \mathbf{1}, \quad n \geq 0 .
$$

The difficulty with $\mathcal{L}$ lies in the fact that $\sigma$ is an expansive transformation.

The operator $L$, adjoint to the operator $\mathcal{L}$ in the duality $L^{1}-L^{\infty}$, is defined on $L^{\infty}([0,1])$ by

$$
\int f \cdot \mathcal{L} g=\int L f \cdot g, \quad g \in L^{\infty}, \quad f \in L^{1} .
$$

In view to get an explicit formula for the $L$-action, we just write

$$
2 \int_{0}^{1} f(t)|\sin \pi t| g(2 t) \mathrm{d} t=\int_{0}^{1 / 2}+\int_{1 / 2}^{1}
$$

for letting the two inverse branches of $\sigma$ into play, and a simple change of variable gives

$$
\int_{0}^{1} g(t)\left(f\left(\frac{t}{2}\right)|\sin \pi t / 2|+f\left(\frac{1+t}{2}\right)|\cos \pi t / 2|\right) \mathrm{d} t=: \int L f \cdot g,
$$

with now

$$
L f(t)=f\left(\frac{t}{2}\right)|\sin \pi t / 2|+f\left(\frac{1+t}{2}\right)|\cos \pi t / 2|=\sum_{\sigma x=t} f(x)|\sin \pi x| .
$$

Observe that $L$ is positive in the lattice sense: $f \geq 0$ implies $L f \geq 0$. Turning back to $u_{n}$, we obtain by duality the following interpretation:

$$
u_{n}=\int_{0}^{1} L^{n} \mathbf{1}=\left\|L^{n} \mathbf{1}\right\|_{L^{1}}
$$




\section{MARTINE QUEFFÉLEC}

The following has been established in [13, 1]:

TheORem 3.1. The $L^{1}$-norms of the TM polynomials

$$
\left\|p_{n}\right\|_{1}=\int_{0}^{1} \prod_{j=0}^{n-1} 2\left|\sin \left(\pi 2^{j} t\right)\right| \mathrm{d} t \approx 2^{n \delta}, \quad \delta=0.40325 \cdots,
$$

where $2^{\delta}$ can be realized as the spectral radius of the Perron-Frobenius operator:

$$
L f(t)=f\left(\frac{t}{2}\right)|\sin \pi t / 2|+f\left(\frac{1+t}{2}\right)|\cos \pi t / 2|
$$

on a suitable Banach space.

Sketch of Proof. We consider a more appropriate Banach space

$$
E=\operatorname{Lip}_{1}(0,1) \quad \text { with the norm } \quad\|f\|=\|f\|_{\infty}+\sup _{x \neq y} \frac{|f(x)-f(y)|}{|x-y|} ;
$$

$L$ is a bounded operator on $E$, not-but almost - compact, more precisely, a quasi-compact operator, whose definition is contained in the following lemma.

Lemma 3.2. There exist a real number $r \geq 0$ and a decomposition of the space $E=D \oplus F$ into invariant closed subspaces with $1 \leq \operatorname{dim} F<\infty$ such that

(i) the spectral radius $r\left(L_{\mid D}\right)<r$;

(ii) the eigenvalues of $L_{\mid F}$ have modulus $\geq r$.

In addition, $L$ being positive in the lattice sense, $L$ is a Perron-Frobenius operator and this implies the following:

Lemma 3.3. The operator $L$ admits a leading, positive eigenvalue $\lambda$, unique with $|\lambda|=r(L)$, which is simple and associated to a non-negative eigenfunction $\varphi \in E$.

Now, by the lemmas, $E=\operatorname{ker}(L-\lambda I) \oplus G$, where $r\left(L_{\mid G}\right)<\lambda$; so we decompose $1=\alpha \varphi+g, g \in G$, and get $L^{n} 1=\alpha \lambda^{n} \varphi+L^{n} g$; but $\left\|L^{n} g\right\|=o\left(\lambda^{n}\right)$ by the dominating property of $\lambda$, and clearly, $\left\|L^{n} g\right\|_{1} \leq\left\|L^{n} g\right\|$; it infers

$$
\left\|L^{n} 1\right\|_{1}=c \lambda^{n}+o\left(\lambda^{n}\right), \quad \text { with } \quad c=\alpha\|\varphi\|_{1},
$$

and the second claim. The first numerical estimates detailed in [13 have been improved in [1]. 


\section{QUESTIONS AROUND THE THUE-MORSE SEQUENCE}

Remark. Actually, $r(L)=\lim _{n \rightarrow \infty}\left\|L^{n} 1\right\|_{\infty}^{1 / n}$. To prove that, denote this limit by $\rho$. Since $\left\|L^{n} 1\right\|_{\infty} \leq\left\|L^{n} 1\right\|$, we clearly have $\rho \leq r(L)$. Now, thanks to the positivity of $L,\left\|L^{n} f\right\|_{\infty} \leq\left\|L^{n} 1\right\|_{\infty}\|f\|_{\infty}$ so that, taking $f=\varphi$ the eigenfunction associated to $\lambda=r(L)$,

$$
r(L)^{n}\|\varphi\|_{\infty}=\left\|L^{n} \varphi\right\|_{\infty} \leq\left\|L^{n} 1\right\|_{\infty}\|\varphi\|_{\infty} \quad \text { and } \quad r(L) \leq \lim _{n \rightarrow \infty}\left\|L^{n} 1\right\|_{\infty}^{1 / n}=: \rho .
$$

\section{The Thue-Morse measure}

W i e n e r introduced in [37] a statistical tool in order to estimate the randomness of some deterministic sequence : the correlation measure. The TM sequence enjoys this remarkable property that the correlation measure exists and can be precisely described. This is once more a consequence of (11) and of the previous polynomial estimates.

\subsection{General facts about correlation measures}

Recall that, for a general bounded complex sequence $\mathbf{u}=\left(u_{n}\right)$, its correlation measure $\sigma_{u}$ (when existing) is the probability measure on $\mathbb{T}$ whose Fourier coefficients are given by

$$
\widehat{\sigma}_{u}(k)=\lim _{N \rightarrow \infty} \frac{1}{N} \sum_{n \leq N} u_{n+k} \overline{u_{n}} \text { if } k \geq 0,
$$

extended to $\mathbb{Z}$ by $\widehat{\sigma}_{u}(-k)=\overline{\widehat{\sigma}_{u}(k)}$ for $k \geq 0$. In other terms, $\sigma_{u}$ is the weak ${ }^{*}$-limit of the sequence of trigonometric polynomials

$$
R_{N}(x)=\frac{1}{N}\left|\sum_{n<N} u_{n} e^{2 \pi i n x}\right|^{2}=: \frac{1}{N}\left|P_{N}\right|^{2}
$$

(assuming $\sigma_{u}$ does exist). Therefore, polynomial estimates are relevant for the analysis of $\sigma_{u}$.

Let $W$ consist of the bounded complex sequences with a unique correlation measure, called the Wiener space. If $u \in W, \sigma_{u}$ is a continuous measure (with no atoms) if and only if $\frac{1}{N} \sum_{0 \leq n<N}\left|\widehat{\sigma}_{u}(n)\right|^{2} \rightarrow 0$.

Proposition 4.1. Let $u \in W$ be a sequence with unimodular terms such that $R_{N}(x) \leq C$ for every $x$; then $\sigma_{u} \ll m$ with bounded Radon-Nykodym derivative $\left|\mathrm{d} \sigma_{\mathrm{u}} / \mathrm{dm}\right| \leq \mathrm{C}$.

This is the case for the Rudin-Shapiro sequence (RS) but also for some so-called generalized Rudin-Shapiro sequences $([2,29])$. 
Proof. Let $\left(F_{j}\right)$ be the sequence of Fejér kernels; then

$$
\left(R_{N} * F_{j}\right)(t)=\sum_{|k|<j}\left(1-\frac{|k|}{j}\right) \widehat{R}_{N}(k) e(k t) ;
$$

moreover,

$$
\left\|R_{N} * F_{j}\right\|_{\infty} \leq\left\|R_{N}\right\|_{\infty} \leq C .
$$

Then, by denoting $\sigma:=\sigma_{u}$,

$$
R_{N} * F_{j} \rightarrow \sigma * F_{j} \text { pointwise as } N \rightarrow \infty,
$$

and in addition,

$$
\left\|\sigma * F_{j}\right\|_{\infty} \leq C
$$

Let now $f \in L^{\infty}$ be a weak ${ }^{*}$-limit point of $\left(\sigma * F_{j}\right)$ in $L^{\infty}$; by testing against a character $e(k t)$, we see that $\hat{f}(k)=\hat{\sigma}(k)$; this infers that the sequence $\left(\sigma * F_{j}\right)$ has a unique weak ${ }^{*}$-limit point, $f \geq 0$, bounded, such that $\sigma=f \mathrm{~d} t$.

Remark. The proposition remains true as soon as $\left\|\sum_{n<N} u_{n} e^{2 \pi i n t}\right\|_{p}=O(\sqrt{N})$ for some $p>2$. But the converse does not hold: if $\sigma_{u}$ is an absolutely continuous measure, one can only assert that the sequence $\left(R_{N}\right)$ is bounded in $L^{1}$-norm.

\subsection{The Thue-Morse (correlation) measure}

It is the probability measure on $\mathbb{T}$ with Fourier coefficients:

$$
\hat{\rho}(k)=\lim _{N \rightarrow \infty} \frac{1}{N} \sum_{n<N} t_{n+k} t_{n} \text { if } k \geq 0,
$$

and symmetric: $\hat{\rho}(-k)=\hat{\rho}(k)$. In a more condensed form,

$$
\rho=\text { weak }^{*}-\lim _{N \rightarrow \infty} \frac{1}{N}\left|P_{N}\right|^{2}=\text { weak }^{*}-\lim _{n \rightarrow \infty} \frac{1}{2^{n}}\left|p_{n}\right|^{2} .
$$

By using again the properties of $\left(t_{n}\right)$ or $\left(p_{n}\right)$,

$$
\rho=\prod_{n \geq 0}\left(1-\cos \left(2 \pi 2^{n} t\right)\right)
$$

but in the weak*-topology, and this characterization of its Fourier coefficients follows $(n \geq 0)$ :

$$
\hat{\rho}(2 n)=\hat{\rho}(n), \quad \hat{\rho}(2 n+1)=-\frac{1}{2}(\hat{\rho}(n)+\hat{\rho}(n+1)), \quad \hat{\rho}(0)=1 .
$$

As a first trivial observation, the image $\hat{\rho}(\mathbb{Z})$ is rational; whence a question of P. S a r nak [31]: does the convolution spectrum of $\rho$, i.e., $\overline{\hat{\rho}(\mathbb{Z})}$ remain countable? 


\section{QUESTIONS AROUND THE THUE-MORSE SEQUENCE}

Moreover, $\rho$ enjoys the following extremal properties [28]:

- $\rho$ is 2-invariant (12).

- $\rho$ is continuous and singular.

- $\rho$ is 2-mixing : $\hat{\rho}\left(2^{n} a+b\right) \rightarrow \hat{\rho}(a) \hat{\rho}(b)$, for all $a \geq 0, b<2^{n}$.

- If $\omega_{n}=\frac{1}{2^{n}} \sum_{k<2^{n}} \delta_{k / 2^{n}}$, then $\rho=q_{n} \cdot \rho * \omega_{n}$ with $q_{n}=\frac{1}{2^{n}}\left|p_{n}\right|^{2}$ (non-dissociated Riesz product).

The behavior of $\hat{\rho}$ along subsequences of $\mathbb{Z}$ is very informative on the arithmetic nature of the support of $\rho$. As for classical Riesz products, we could expect that $\hat{\rho}$ is sensitive to the 2 -sum of digits, maybe in a more intricate way, but this would help for the answer because of what follows.

The representations of an integer in both bases $r$ and $s$, when $r$ and $s$ are multiplicatively independent (i.e., $\log r / \log s \notin \mathbb{Q}$ ), have already been studied and compared: the following result appears as a consequence of a key result on Bernoulli convolution measures.

Theorem 4.1 (Senge \& Straus). The number of integers, the sum of whose digits in each of two bases $r$ and $s$ lies below a fixed bound, is finite if and only if $\log r / \log s$ is irrational.

In particular, since $S_{3}\left(3^{k}\right)=1, S_{2}\left(3^{k}\right)$ cannot be bounded. A few years later, $\mathrm{S}$ t e w a r t gave a quantitative version of Senge and Straus' theorem:

ThEOREM 4.2 (Stewart). If $r$ and $s$ are multiplicatively independent, there exists $d>0$, such that for every $c$, each $m>25$ with $\max \left(S_{r}(m), S_{s}(m)\right)<c$ satisfies

$$
\frac{\log \log m}{\log \log \log m+d}<2 c+1 \text {. }
$$

Actually, by looking at the coefficients $\hat{\rho}\left(2^{k}-1\right)$, we guess that another digital function should be more pertinent for our problem, which counts the number of times a digit different from the previous one is read.

Definition 4.1. We denote by $D C(n, q)$ the number of changes of digits in the writing of $n$ to base $q$.

These two functions $D C$ and $S$ are related and can easily be compared since, clearly,

$$
D C(n, q) \leq 2 S_{q}(n)+1
$$

but they are quite different on blocks of identical digits $\neq 0$, thus non-equivalent. The following property of $\rho$ has been established by K a m a e [20]

TheOREM 4.3. There exists $0<\delta<1$ such that $\hat{\rho}(n) \ll \delta^{D C(n, 2)}$; 


\section{MARTINE QUEFFÉLEC}

K a m a e referred for that to the TM dynamical system viewed as an extension of the 2-odometer by a cocycle [16, 21].

In 2013, Bugeaud, Cipu and Mignotte proposed a $D C$-version of Stewart's result, which, by the remarks above, provides an improvement of it.

Theorem 4.4 (B u g e a u d, C i p u \& Mignotte 2013). If $r$ and $s$ are multiplicatively independent, there exists $C$ such that

$$
D C(m, r)+D C(m, s) \geq \frac{\log \log m}{\log \log \log m+C}-1 \quad \text { for } m>25 .
$$

Combining these two last theorems, we deduce

$$
\hat{\rho}\left(3^{n}\right) \ll \delta^{\frac{\log n}{\log \log n+K}}
$$

for some constant $K>0$; also, if $n>m$, since $D C\left(3^{n}-3^{m}, 3\right)=1$,

$$
\hat{\rho}\left(3^{n}-3^{m}\right) \ll \delta^{\frac{\log n}{\log \log n+K}} .
$$

\subsection{Analysis of singular measures}

Different parameters make it possible to classify singular measures according to their "size" and some of them are linked to the Fourier coefficients (a good reference for that is [12]; see also [38]). The sought-after goal is to compare the given measure with the Hausdorff family of outer measures $\left(\mathcal{H}^{\alpha}\right)$.

Definition 4.2. A measure $\mu$ on $\mathbb{R}\left(\right.$ or $\left.\mathbb{R}^{d}\right)$ is $\alpha$-Hölder with $\alpha \geq 0$ if there exists $C>0$ such that

$$
\mu(B(x, r)) \leq C r^{\alpha}, \text { for all } x, \text { for all } 0 \leq r \leq 1 .
$$

(In fractal geometry $\mu$ is called a Frostman measure). We put

$$
\alpha(\mu)=\inf \{\alpha \text { such that (13) holds }\} .
$$

A deeper analysis consist in studying the local exponents $\alpha(x)$ defined by

$$
\mu(B(x, r)) \sim C r^{\alpha(x)}
$$

when $r \rightarrow 0$ and, for any given $\alpha>0$,

$$
E_{\alpha}=\{x, \alpha(x)=\alpha\}
$$

this leads to the multifractal analysis of the measure by computing $\operatorname{dim} E_{\alpha}$. From now on, we restrict ourselves to $[0,1)$ (identified with $\mathbb{T}$ ). 


\section{QUESTIONS AROUND THE THUE-MORSE SEQUENCE}

\subsubsection{Energy}

The exponent coming out in the $L^{4}$-norms has some meaning in terms of the TM correlation measure as we shall see. What about the $L^{2 k}$-norms?

The energy represents in some sense a mean value of the local exponents; this notion originally comes from potential theory.

Definition 4.3. Let $\mu$ be some probability measure on $[0,1)$; we consider

$$
I_{\beta}(\mu):=\iint \frac{\mathrm{d} \mu(\mathrm{x}) \mathrm{d} \mu(\mathrm{y})}{|\mathrm{x}-\mathrm{y}|^{\beta}} ;
$$

the energy exponent (or capacity) of $\mu$ is

$$
e(\mu)=\sup \left\{0 \leq \beta \leq 1 ; I_{\beta}(\mu)<\infty\right\}
$$

Before explaining the role it will play, it is convenient to give another expression of $e(\mu)$ in terms of the Fourier coefficients of $\mu$.

Proposition 4.2. We have

$$
\begin{aligned}
e(\mu) & =\sup \left\{0 \leq \alpha<1 ; \sum_{\mathbb{Z}^{*}}|\hat{\mu}(n)|^{2}|n|^{\alpha-1}<+\infty\right\} \\
& =1-\inf \left\{0 \leq \beta<1 ; \lim \sup N^{-\beta} \sum_{|n|<N}|\hat{\mu}(n)|^{2}<+\infty\right\} .
\end{aligned}
$$

We retain that, if some $0 \leq \delta \leq 1$ exists such that $\frac{1}{N} \sum_{|n|<N}|\hat{\mu}(n)|^{2}=O\left(N^{-\delta}\right)$, then $e(\mu) \geq \delta$. An old result of Wiener can be revisited in that way (see [17]):

Proposition 4.3. For every probability measure on $[0,1)$,

$$
e(\mu) \geq \alpha(\mu)
$$

\subsubsection{Dimension of measure}

When $\mu$ is a singular probability measure, we take the liberty to speak of "the" Borel support of $\mu$ although it is far from being unique when $\mu$ is continuous! Such a support being negligible, we are reduced to evaluate its Hausdorff dimension and therefore the size of the measure:

Definition 4.4. The following exponent is called: dimension of the measure $\mu$ and we denote it by $\mathrm{d}(\mu)([12])$ :

$$
\mathrm{d}(\mu)=\inf \left\{\operatorname{dim}_{\mathrm{H}}(\mathrm{E}) ; \mu(\mathrm{E})=1\right\} .
$$




\section{MARTINE QUEFFÉLEC}

Proposition 4.4. For every probability measure on $[0,1)$,

$$
\mathrm{d}(\mu) \geq \mathrm{e}(\mu) .
$$

Remark. In the general case, those parameters are distinct, but they coincide for self-similar measures, such as the Cantor-Lebesgue measure, for example. What about the TM measure?

\subsubsection{Entropy}

The last two parameters we mention are available in a dynamical context, with $\mu$ an invariant (and ergodic) probability measure for some transformation $T$ of the interval (a good reference is [26]).

1. The entropy of $\mu$; in the case of a Markov shift (with $\left(P_{n}\right)$ as generating partitions of intervals), it can be recovered (defined), with the help of the ShannonMacMillan theorem, as an almost everywhere limit:

$$
h(\mu):=\lim _{n \rightarrow \infty} \frac{1}{n} \log \mu\left(I_{n}(x)\right) \text { a.e., }
$$

where $I_{n}(x)$ is the element of $P_{n}$ containing $x$.

When, in addition, the transformation $T$ is an expanding $C^{1}$-piecewise transformation of the interval, we have at our disposal an auxiliary tool:

2. The Lyapounov exponent $\lambda=\lambda(\mu)=\lim _{n \rightarrow \infty} \frac{1}{n} \int \log \left(T^{n}\right)^{\prime} \mathrm{d} \mu$, which measures the mean ratio of dilation.

A very useful formula due to Young 39 connects these quantities in the latter case:

Proposition 4.5. In these "good" cases, $h(\mu)=\mathrm{d}(\mu) \lambda(\mu)$.

\subsection{Back to $\rho$}

We try to compute those parameters for the measure $\rho$. Many things are well-known for self-similar measures, but $\rho$ is not self-similar... However it is interesting to determine what remains despite that for this very simple measure.

First of all, its closed support (the smaller closed set with negligible complementary) is the whole circle contrary to canonical self-similar measures. This is a consequence of the famous Rokhlin's lemma.

\subsection{1. $e(\rho)$ and $h(\rho)$}

We are able to describe two of these parameters for $\rho$ :

1. The energy exponent $e(\rho)$ can be computed by using proposition 4.2 and the 


\section{QUESTIONS AROUND THE THUE-MORSE SEQUENCE}

description (12) of the coefficients $\hat{\rho}(n)$; we proceed exactly as for the $L^{4}$-norm to get:

$$
\sum_{|n|<N}|\hat{\rho}(n)|^{2} \approx N^{\gamma}
$$

with $\gamma=\log _{2} \kappa$ when $N$ is some power of 2 ; it follows that

$$
\inf \left\{0 \leq \beta<1 ; \quad \limsup _{N \rightarrow \infty} N^{-\beta} \sum_{|n|<N}|\hat{\rho}(n)|^{2}<+\infty\right\} \geq \gamma
$$

since, for $\beta<\gamma$,

$$
\limsup _{N \rightarrow \infty} N^{-\beta} \sum_{|n|<N}|\hat{\rho}(n)|^{2} \geq \limsup _{N \rightarrow \infty} 2^{-n \beta} \sum_{|k|<2^{n}}|\hat{\rho}(k)|^{2}=\infty .
$$

For the upper bound, $N$ being arbitrary, say $2^{K} \leq N<2^{K+1}$, we have $N^{\gamma} \geq 2^{K \gamma}$ and

$$
N^{-\gamma} \sum_{|n|<N}|\hat{\rho}(n)|^{2} \leq 2^{-K \gamma} \sum_{|k|<2^{K+1}}|\hat{\rho}(k)|^{2}=2^{\gamma} 2^{-(K+1) \gamma} \sum_{|k|<2^{K+1}}|\hat{\rho}(k)|^{2} ;
$$

thus

$$
\limsup _{N \rightarrow \infty} N^{-\gamma} \sum_{|n|<N}|\hat{\rho}(n)|^{2}<\infty
$$

and

$$
\inf \left\{0 \leq \beta<1 ; \limsup _{N \rightarrow \infty} N^{-\beta} \sum_{|n|<N}|\hat{\rho}(n)|^{2}<+\infty\right\} \leq \gamma .
$$

Finally, $e(\rho)=1-\log _{2} \kappa \sim 0.643$, where $\kappa$ appeared in (7).

2. We can compute: $h(\rho)=\log 2-\int \log (1-\cos 2 \pi x) \mathrm{d} \rho(\mathrm{x})$ whence an implicit expression of $d(\rho)$ :

$$
\mathrm{d}(\rho)=1-\int \log _{2}(1-\cos 2 \pi \mathrm{x}) \mathrm{d} \rho(\mathrm{x}),
$$

according to Young's identity.

P r o o f. Actually, there is a general result for $q$-invariant measures thanks to the Kolmogorov-Sinaï theorem (see [18]). In our case (not so specific by the way) let us start with the generalized Riesz product property of $\rho$ :

$$
\rho=q_{n} \rho_{n}:=q_{n} \cdot \rho * \omega_{n}, \text { where } q_{n}=2^{-n}\left|p_{n}\right|^{2},
$$

and $\omega_{n}$ is the Haar measure of the group of dyadic rational numbers $\left\{k / 2^{n}\right\}$. From the cocycle equation

$$
q_{n}(x)=q_{1}(x) q_{1}(2 x) \cdots q_{1}\left(2^{n-1} x\right) \quad \text { with } \quad q_{1}(x)=(1-\cos 2 \pi x),
$$




\section{MARTINE QUEFFÉLEC}

we can deduce that for $\rho$-almost all $x$,

$$
\lim _{n \rightarrow \infty} \frac{1}{n} \log q_{n}(x)=\lim _{n \rightarrow \infty} \frac{1}{n} \sum_{j<n} \log q_{1}\left(2^{j} x\right)=\int q_{1}(x) \mathrm{d} \rho(\mathrm{x})=: \alpha,
$$

since $\rho$ is a 2-ergodic measure. For such a generic point $x$ and $n \geq 1$, let $I_{n}(x)=\left[j(x) 2^{-n},(j(x)+1) 2^{-n}\right.$ [ be the interval of the partition $P_{n}=\left\{\left[j 2^{-n}\right.\right.$, $(j+1) 2^{-n}\left[, 0 \leq j \leq 2^{n}-1\right\}$, containing $x$. By the above limit, for $\varepsilon>0$, there exists $N(\varepsilon)$ such that, if $n \geq N(\varepsilon)$ and $t \in I_{n}(x)$,

so that

$$
e^{n(\alpha-\varepsilon)} \leq q_{n}(t) \leq e^{n(\alpha+\varepsilon)}
$$

$$
e^{n(\alpha-\varepsilon)} \rho_{n}\left(I_{n}(x)\right) \leq \rho\left(I_{n}(x)\right) \leq e^{n(\alpha+\varepsilon)} \rho_{n}\left(I_{n}(x)\right) .
$$

Now, by definition of $\rho_{n}$ and putting $I:=I_{n}(x)$, we have

$$
\rho_{n}(I)=\frac{1}{2^{n}} \sum_{k=0}^{2^{n}-1} \int \mathbf{1}_{I}\left(t+\frac{k}{2^{n}}\right) \mathrm{d} \rho(\mathrm{t})=\frac{1}{2^{\mathrm{n}}} \int \sum_{\mathrm{k}=0}^{2^{\mathrm{n}}-1} \mathbf{1}_{\mathrm{I}+\frac{\mathrm{k}}{2^{\mathrm{n}}}}(\mathrm{t}) \mathrm{d} \rho(\mathrm{t})=\frac{1}{2^{\mathrm{n}}} ;
$$

Finally, we have proved that

$$
\lim _{n \rightarrow \infty} \frac{1}{n} \log \rho\left(I_{n}(x)\right)=\alpha-\log 2, \quad \text { as expected. }
$$

Remark. This implicit formula for $d(\rho)$ is not so convenient and does not allow to answer a question of Vershik on the value of $\mathrm{d}(\rho)$ ? Note the equivalent formulation: $\mathrm{d}(\rho)=-\frac{1}{\log 2} \sum_{\mathrm{j} \neq 0} \hat{\rho}(\mathrm{j}) /|\mathrm{j}|$, quite as implicit, but it seems to infer $\mathrm{d}(\rho)>\mathrm{e}(\rho)$ ?

\subsubsection{Multifractal analysis}

We do expect something close to fractal regularity for the TM measure; numerical estimations have been carried out $([15,3])$ and let think that the Hölder exponent $\alpha(\rho)=\inf \{\alpha(x), x \in[0,1]\}=2-\frac{\log 3}{\log 2} \sim 0.415037$. The multifractal study of $\rho$ has to be completed.

\subsubsection{Borel supports of $\rho$}

A description of Borel sets charged by $\rho$ remains an interesting problem. It seems quite natural, for a $q$-invariant measure, to set the question of support in terms of distribution mod 1 of reals numbers; indeed, a first elementary observation is the following one: any q-invariant, ergodic and non absolutely continuous probability measure (thus singular) $\mu$ does not charge the set of $q$-normal numbers. Let us recall some definitions. 


\section{QUESTIONS AROUND THE THUE-MORSE SEQUENCE}

Definition 4.5. The real number $x$ is $q$-normal if $\left\{q^{n} x\right\}$ is uniformly distributed. If $\Lambda=\left\{n_{k}\right\}$ is some increasing sequence of integers, $x$ is $\Lambda$-normal if $\left\{n_{k} x\right\}$ is uniformly distributed.

If $\mu \neq m$ the Lebesgue measure on $\mathbb{T}$, by choosing $k$ with $\hat{\mu}(k) \neq 0$ and using the ergodic theorem, we get indeed

$$
\frac{1}{N} \sum_{n<N} e\left(k q^{n} x\right) \rightarrow \hat{\mu}(k) \neq 0 \mu-\text { a.e. }
$$

while the lefthandside tends to 0 if $x$ is $q$-normal (Weyl criterion); so $\mu$-almost no $x$ is $q$-normal.

After Cass els has proved that the Cantor-Lebesgue measure supports the set of 2-normal numbers (which are not 3-normal) [5], the analogous question arose for the TM measure and the set of 3-normal numbers. K a m a e [20] proved in turn:

TheOREM 4.5. $\rho$-almost all numbers are normal to base 3 , i.e., $\rho$ charges the set of 3-normal numbers which are not 2-normal.

The proof rests on a classical and very efficient technique (8]) that $\mathrm{C}$ as sels has already used; it reduces the proof to the following:

LEMma 4.1. For every $k \neq 0$,

$$
\left.\sum_{N} \frac{1}{N^{3}} \int \mid \sum_{n<N} e\left(3^{n} k x\right)\right)\left.\right|^{2} \mathrm{~d} \rho(\mathrm{x})<\infty
$$

Sketch of proof. By expanding the square of the sum, we are led to estimate quantities such as $\hat{\rho}\left(k\left(3^{n}-3^{m}\right)\right)$. By theorem 4.3, we see that, $k$ being fixed, there exists $\varepsilon>0$ such that

$$
\sum_{1 \leq n, m \leq N} \hat{\rho}\left(\left(3^{n}-3^{m}\right) k\right)=O\left(\frac{N^{2}}{(\log N)^{1+\varepsilon}}\right)
$$

whence the lemma and the theorem.

In the same period, W. Schmidt constructed a measure supporting $r$ normal but non $s$-normal numbers, for multiplicatively independent $r$ et $s$; Cassels' and Kamae's theorems have the advantage to provide a natural example of such a measure.

Let us recall a more general result of $\mathrm{B}$. H o s t [18. 


\section{MARTINE QUEFFÉLEC}

TheOREM 4.6. Let $p$ et $q$ be two integers $\geq 2$ and coprime, and let $\mu$ be a $p$-invariant and p-ergodic probability measure with positive entropy (for the $p$ -shift). Then $\mu$-almost all number is q-normal.

Remark. If we denote by $W_{\alpha}$ the set of $\alpha$-approximable numbers e.g. the numbers with irrationality exponent greater than or equal $\alpha, \mathcal{W}=\cup_{\alpha>2} W_{\alpha}$ is the set of well-approximable numbers. B. Weiss [36] has proved that $\mu(W)=0$ for the Cantor-Lebesgue measure $\mu$.

Though it is far from evident, could we explore the support of $\rho$ in a similar arithmetical way?

\section{Back to sequences}

We intend to deduce some information on the initial sequence from its correlation measure, and, by the way, to come full circle in our survey. The following elementary fact is somewhat promising. If $u \in W$,

$$
\lim _{N \rightarrow \infty} \frac{1}{N} \sum_{n \leq N} u_{n} e(n \alpha)=0 \Longleftrightarrow \sigma_{u}\{\alpha\}=0 .
$$

We could expect something better indeed for the TM sequence.

\subsection{Affinity of measures}

The notion of disjoint dynamical systems has been introduced by $\mathrm{F}$ u r s t e nb er g (1967), in terms of coupling of measures, and a stronger property is the spectral disjointness of systems, which means that the maximal spectral types of those systems are mutually singular. How to detect this property?

Definition 5.1. Let $\mu$ and $\nu$ be two positive measures in $M(\mathbf{T})$ and $\lambda \in M(\mathbf{T})$ be such that both $\mu \ll \lambda$ and $\nu \ll \lambda$. The affinity $\rho(\mu, \nu)$ of the measures $\mu$ and $\nu$ is the quantity

$$
\rho(\mu, \nu)=\int_{\mathbf{T}}\left(\frac{\mathrm{d} \mu}{\mathrm{d} \lambda}\right)^{1 / 2}\left(\frac{\mathrm{d} \nu}{\mathrm{d} \lambda}\right)^{1 / 2} \mathrm{~d} \lambda,
$$

obviously independent of the choice of $\lambda$.

Note that

$$
\rho(\mu, \nu)=0 \text { if and only if } \mu \perp \nu .
$$

We can say a little bit more ... if we are aware of the following proposition: 


\section{QUESTIONS AROUND THE THUE-MORSE SEQUENCE}

Proposition 5.1. Let $\left(\mu_{n}\right)$ and $\left(\nu_{n}\right)$ be two sequences of positive measures on $\mathbf{T}$ such that $\mu_{n} \rightarrow \mu$ and $\nu_{n} \rightarrow \nu$ in the weak-star topology of $M(\mathbf{T})$. Then

$$
\limsup _{n \rightarrow \infty} \rho\left(\mu_{n}, \nu_{n}\right) \leq \rho(\mu, \nu),
$$

where $\rho$ denotes the affinity defined in (15)

Applied with correlation measures, this provides a weak form of disjunction for the systems generated by sequences.

Proposition 5.2. Let $u$ and $v$ be two bounded sequences in the Wiener space with $\sigma_{u}$ and $\sigma_{v}$ as their correlation measures. Then,

$$
\limsup _{N \rightarrow \infty} \frac{1}{N}\left|\sum_{n<N} u_{n} \overline{v_{n}}\right| \leq \rho\left(\sigma_{u}, \sigma_{v}\right) .
$$

In particular, $\sigma_{u} \perp \sigma_{v}$ implies

$$
\frac{1}{N} \sum_{n<N} u_{n} \overline{v_{n}} \rightarrow 0
$$

By extension, we say that the sequences $u$ and $v$ are orthogonal and note $u \perp v$ if (16) holds. As a consequence, if $\sigma_{u}$ is a singular measure and $R_{N}$ is given by (11),

$$
\int_{\mathbb{T}}\left(R_{N}\right)^{1 / 2} 1 \mathrm{~d} t=\int_{\mathbb{T}} \frac{1}{\sqrt{N}}\left|\sum_{n<N} u_{n} e^{2 \pi i n t}\right| \mathrm{d} t \rightarrow 0
$$

when $N \rightarrow \infty$, i.e.,

$$
\left\|P_{N}\right\|_{1}=o(\sqrt{N})
$$

And $\sigma_{u}$ is a continuous measure if and only if $\lim _{N \rightarrow \infty} \frac{1}{N} \sum_{n<N} u_{n} e(n x)=0$ for every $x$, but when does it hold uniformly in $x$ ?

EXAMPLE. The TM sequence $(-1)^{S_{2}(n)}$ and $(-1)^{S_{3}(n)}$ are orthogonal; so are the $T M$ and the RS sequences, whence $\frac{1}{N} \sum_{n<N}(-1)^{\# 1(n)}(-1)^{\# 11(n)} \rightarrow 0$, since the Rudin-Shapiro sequence counts the number of patterns 11 in the representation of $n$.

A famous example has been extensively studied these last years: the Moebius sequence $(\underline{32})$. 


\subsection{Moebius' orthogonality and Sarnak's conjecture}

Let us recall:

Definition 5.2. The Moebius function $\mu$ is so defined: $\mu(1)=1$ and

$$
\mu(n)=\left\{\begin{array}{lll}
(-1)^{r} & \text { if } n=p_{1} p_{2} \cdots p_{r}, p_{j} \text { distinct } \\
0 & \text { if } n \text { has a square factor. }
\end{array}\right.
$$

We summarize in a few lines the important properties of the function $\mu$ in the spirit of our survey. We must keep in mind the following conjecture: the correlation measure of the Moebius sequence is absolutely continuous with respect to the Lebesgue measure.

1. The Prime Number Theorem is equivalent to:

$$
\sum_{n \leq N} \mu(n)=o(N)
$$

2. The Riemann hypothesis is equivalent to the estimate:

$$
\forall \varepsilon>0, \quad \sum_{n \leq N} \mu(n)=O_{\varepsilon}\left(N^{1 / 2+\varepsilon}\right) .
$$

3. Davenport has proved:

Theorem 5.1. For every $A \geq 1$, there exists some constant $C$ such that

$$
\sup _{x \in[0,1]}\left|\sum_{n \leq N} \mu(n) e(n x)\right| \leq C \frac{N}{(\log N)^{A}}
$$

4. Under Riemann Hypothesis:

Theorem 5.2 (Huxley \& Ramaré). For every $\varepsilon>0$ there exists some constant $C$ such that

$$
\sup _{x \in[0,1]}\left|\sum_{n \leq N} \mu(n) e(n x)\right| \leq C N^{3 / 4+\varepsilon}
$$

while $1 / 2$ is the expected exponent.

In terms of disjointness, the assertion 1 . means that $\mu \perp \mathbf{1}$ and could be interpreted as: the correlation of $\mu$ has no point mass on 0 .

The estimate in 2. means $\frac{1}{N}\left|\sum_{n \leq N} \mu(n)\right|^{2}=O\left(N^{\varepsilon}\right)$ and is of no help in tackling the conjectured property of $\mu$. Does Davenport's result imply that $\sigma_{\mu}$ is a continuous measure and nothing more ...?

A pioneer result on Moebius' orthogonality is at the origin of many works $([9,23])$. 


\section{QUESTIONS AROUND THE THUE-MORSE SEQUENCE}

Theorem 5.3 (Dartyge-Tenenbaum, Mauduit-Rivat). The TM sequence is orthogonal to the Moebius one: $\sum_{n \leq N} t_{n} \mu(n)=o(N)$.

Of course, this could be derived from the pre-supposed absolute continuity of $\sigma_{\mu}$. This orthogonality property has been enlarged to many sequences (or systems) with low complexity, with the final conjecture formulated by $\mathrm{S}$ a r n a k 32 . as a focal point, asserting that:

SARnAK's ConjeCtuRe. For any deterministic (with 0-entropy) dynamical system $(X, T)$,

$$
\sum_{n<N} \mu(n) f\left(T^{n} x\right)=o(N) \quad \text { for every } \quad f \in C(X) \quad \text { and } \quad x \in X .
$$

\section{Recapitulating questions}

We collect in this last section different questions arising from this survey, some of them seeming accessible ...

- What is the $\lim _{N \rightarrow \infty} \frac{1}{N} \sum_{n<N} t_{3^{n}}$ ?

- The uniform bound of the TM polynomials is attained at a 2-periodic cycle for the 2-shift: $\{1 / 3,2 / 3\}$; what about the uniform bound of the polynomials associated to $\left(e^{2 i \pi \alpha S_{2}(n)}\right)$ for some $\alpha \in \mathbb{Q}$ ?

- Can we estimate $\left\|p_{n}\right\|_{2 k}$ for any $k \geq 1$ ?

- Let $\left(\epsilon_{n}\right)$ be the Rudin-Shapiro sequence and $P_{n}(t)=\sum_{0 \leq k<2^{n}} \epsilon_{k} e(k t)$. Prove that for every $q>0$

$$
\int_{\mathbb{T}}\left(\frac{\left|P_{n}(t)\right|^{2}}{2^{n}}\right)^{q} \mathrm{~d} t \rightarrow \frac{1}{2} \int_{0}^{2} x^{q} \mathrm{~d} x=\frac{2^{q}}{q+1}
$$

(see [10] for an approach, [30] for a solution).

- Does the convolution spectrum of $\rho$, i.e., $\overline{\hat{\rho}(\mathbb{Z})}$, remain countable?

- Is there a closed form for $\mathrm{d}(\rho)$ to be compared with $e(\rho)$ ?

- Could we compute the energy exponent for other $q$-multiplicative sequences?

- Achieve the multifractal study of $\rho$ and of $\sigma_{u}$ for a $q$-multiplicative sequence $u$ ?

- Can we identify the Walsh-Fourier coefficients of $\rho: \int_{\mathbb{T}} w_{A}(x) \mathrm{d} \rho(\mathrm{x})$ ? (See [27], for example.)

- (Fürstenberg's conjecture) Every continuous both 2- and 3- invariant probability measure must be Lebesgue?

Acknowledgment The author is very grateful to the referee for his pertinent suggestions and improvements. 


\title{
MARTINE QUEFFÉLEC
}

\author{
REFERENCES
}

[1] AistLEITNER, C.-HOFFER, R.-LARCHER, V.: On evil Kronecker sequences and lacunary trigonometric products, Ann. Inst. Fourier (Grenoble) 67 (2017), 637-687.

[2] AlloUChE, J.P. -LIARDET, P.: Generalized Rudin-Shapiro sequences, Acta Arith. 60 (1991), 1-27.

[3] BAI, Z. Q.: Multifractal analysis of the spectral measure of the Thue-Morse sequence: a periodic orbit approach, J. Phys. A: Math. Gen. 39 (2006), 1959-1973.

[4] BUGEAUD, Y-CIPU, M.-MIGNOTTE, M.: On the representation of Fibonacci and Lucas numbers in an integer base, Ann. Math. Qu. 37 (2013), 31-43.

[5] CASSELS, J.W.S.: On a problem of Steinhaus about normal numbers, Colloq. Math. 7 (1959), 95-101.

[6] COQUET, J.: A summation formula related to the binary digits, Invent. Math. 73 (1983), $107-115$.

[7] DAVEnPORT, H.: On certain exponential sums, J. Reine Angew. Math. 169 (1933), 158-176.

[8] DAVENPORT, H.-ERDŐS, P.-LEVEQUE, W. J.: On Weyl's criterion for uniform distribution, Michigan Math. J. 10 (1963), 311-314.

[9] DARTYGe, C.-TEnenBAUM, G.: Sommes des chiffres de multiples d'entiers, Ann. Inst. Fourier (Grenoble) 55 (2005), 2423-2474.

[10] DOCHE, C.-HABSIEGER, L.: Moments of the Rudin-Shapiro polynomials, J. Fourier Anal. Appl. 10 (2004), 497-505.

[11] DUMOnT, J. M.: Discrépance des progressions arithmétiques dans la suite de Morse, C. R. Acad. Sci. Paris, SŽr. I 297 (1983), 145-148.

[12] FALCONER, K.: Fractal geometry, mathematical foundations and applications, John Wiley \& sons, 1990.

[13] FOUVRY, E.-MAUDUIT, C.: Sommes des chiffres et nombres presque premiers, Math. Ann. 305 (1996), 571-599.

[14] GELFOND, A. O.: Sur les nombres qui ont des propriétés additives et multiplicatives données, Acta Arith. 13 (1968), 259-265.

[15] GODRÈCHE, C.-LUCK, J. M.: Multifractal analysis in reciprocal space and the nature of the Fourier transform of self-similar structures. J. Phys. A 23 (1990), 3769-3797.

[16] GRABNER, P. J.-LIARDET, P.-TICHY, R. F.: Spectral disjointness of dynamical systems related to some arithmetic functions. Publ. Math. Debrecen 66 (2005), 213-243.

[17] HARE, K.-ROGINSKAYA, M.: A Fourier series formula for energy of measures with applications to Riesz products. Proc. Amer. Math. Soc. 131 (2003), 165-174.

[18] HOST, B.: Nombres normaux, entropie, translations, Israel J. Math. 91 (1995), no. 1-3, 419-428.

[19] KAMAE, T.: Cyclic extensions of odometer transformations and spectral disjointness, Israel J. Math. 59 (1987), 41-63.

[20] Number-theoretic problems involving two independent bases. In: Number theory and cryptography (Sydney, 1989), London Math. Soc. Lecture Note Ser., Vol. 154, Cambridge Univ. Press, Cambridge, 1990, pp. 196-203.

[21] LIARDET, P.: Propriétés génériques de processus croisés. Israel J. Math. 39 (1981), 303-325.

[22] MAUDUIT, C.: Multiplicative properties of the Thue-Morse sequence, Period. Math. Hungar. 43 (2001), 137-153. 


\section{QUESTIONS AROUND THE THUE-MORSE SEQUENCE}

[23] MAUDUIT, C.-RIVAT, J.: Sur un problème de Gelfond: la somme des chiffres des nombres premiers, Ann. of Math.(2) 171 (2010), no. 3, 1591-1646.

[24] La somme des chiffres des carrés, Acta Mathematica 203 (2009), 107-148.

[25] NEWMAN, D. J.: On the number of binary digits in a multiple of three, Proc. Amer. Math. Soc. 21 (1969), 719-721.

[26] PESIN, Y.: Dimension theory in dynamical systems, The university of Chicago Press, 1997.

[27] PARREAU, F.-QUEFFÉLEC, M.: Mo measures for the Walsh system, J. Fourier Anal. Appl. 15 (2009), 502-514.

[28] QUEFFÉLEC, M.: Substitution dynamical systems-spectral analysis. (2nd edition.) In: Lecture Notes in Mathematics, Vol. 1294. Springer-Verlag, Berlin, 2010.

[29] Une nouvelle propriété des suites de Rudin-Shapiro. Ann. Inst. Fourier (Grenoble) 37 (1987), 115-138.

[30] RODGERS, B.: On the distribution of Rudin-Shapiro polynomials and lacunary walks on $S U(2)$, Preprint.

https://arxiv.org/pdf/1606.01637

[31] SARNAK, P.: Spectra of singular measures as multipliers on Lp. J. Funct. Anal. 37 (1980), 302-317.

[32] _ Möbius randomness and dynamics, Not. S. Afr. Math. Soc. 43 (2012), 89-97.

[33] SEnGE, H. G.-STRAUS, E. G.: PV -numbers and sets of multiplicity, Period. Math. Hungar. 3 (1973), 93-100.

[34] STEWART, C.L.: On the representation of an integer in two different bases, J. Reine Angew. Math. 319 (1980), 63-72.

[35] TENENBAUM, G.: Sur la non dérivabilité de fonctions périodiques associées à certaines formules sommatoires In: The mathematics of Paul Erdős, I, Algorithms Combin. Vol. 13, Springer-Verlag, Berlin, 1997. pp. 117-128,

[36] WEISS, B.: Almost no points on a Cantor set are very well approximable, R. Soc. Lond. Proc. Ser. A 457 (2001), no. 2008, 949-952.

[37] WIENER, N.: Generalized harmonic analysis, Acta Math. 55 (1930), 117-258.

[38] WOLFF, T. H.: Lectures on Harmonic Analysis, University Lecture Series, Vol. 29. AMS, Providence, RI, 2003.

[39] YOUNG, L.S.: Dimension, entropy and Lyapunov exponents. Erg. Theory Dyn. Syst. 2 (1982), 109-124.

Received June 6, 2016

Accepted March 1, 2017
Martine Queffélec

Laboratoire Paul Painlevé

University Lille 1

59655 Villeneuve d'Ascq cedex

FRANCE

E-mail: martine.queffelec@univ-lille1.fr 